\title{
Best Practice Update: Incorporating Psychogastroenterology Into Management of Digestive Disorders
}

\author{
Laurie Keefer, ${ }^{1}$ Olafur S. Palsson, ${ }^{2}$ and John E. Pandolfino ${ }^{3}$ \\ ${ }^{1}$ Division of Gastroenterology, Icahn School of Medicine at Mount Sinai, New York, New York; ${ }^{2}$ University of North Carolina \\ Chapel Hill, North Carolina; and ${ }^{3}$ Northwestern University Feinberg School of Medicine, Chicago, Illinois
}

\begin{abstract}
Chronic digestive diseases, including irritable bowel syndrome, gastroesophageal reflux disease, and inflammatory bowel diseases, cannot be disentangled from their psychological context-the substantial burden of these diseases is co-determined by symptom and disease severity and the ability of patients to cope with their symptoms without significant interruption to daily life. The growing field of psychogastroenterology focuses on the application of scientifically based psychological principles and techniques to the alleviation of digestive symptoms. In this Clinical Practice Update, we describe the structure and efficacy of 2 major classes of psychotherapy-cognitive behavior therapy and gut-directed hypnotherapy. We focus on the impact of these brain-gut psychotherapies on gastrointestinal symptoms, as well as their ability to facilitate improved coping, resilience, and self-regulation. The importance of the gastroenterologist in the promotion of integrated psychological care cannot be overstated, and recommendations are provided on how to address psychological issues and make an effective referral for brain-gut psychotherapy in routine practice.
\end{abstract}

Keywords: Brain-gut Psychotherapy; GI Psychologist; Gut-directed hypnotherapy; Cognitive Behavior Therapy.

$\mathrm{C}$ hronic digestive disorders cost the health care system billions of dollars and are associated with substantial disease burden. ${ }^{1}$ The burden of these diseases is co-determined by symptom/disease severity and the ability of patients to cope with their symptoms without significant interruption to daily life. In other words, chronic digestive diseases cannot be disentangled from their psychosocial context. The most common chronic digestive disorders are the functional gastrointestinal (GI) and motility disorders, the burdens of which are amplified when symptoms are severe or refractory, psychiatric comorbidity is present, and/or coping skills are impaired. ${ }^{2}$ Patients with reflux hypersensitivity and functional heartburn acquire risk from the unnecessary long-term use of PPIs when esophageal hypervigilance overshadows symptom-reflux correlation. ${ }^{3,4}$ Irritable bowel syndrome (IBS) can progress into severely disabling centrally mediated abdominal pain syndrome ${ }^{5}$; and psychosocial factors, when coupled with opiate use, increase risk of narcotic bowel syndrome. ${ }^{6,7}$ The burden of "organic" conditions, such as Crohn's disease and ulcerative colitis, is similarly amplified by psychosocial factors and poor coping. Indeed, $15 \%$ of patients with inflammatory bowel disease (IBD) account for approximately 50\% of health care expenditures, which appears to be driven by concomitant chronic pain, depression, and poor social support. ${ }^{8,9}$ Depression, when present in IBD, has been shown to increase risk for surgery, hospitalizations, and disability, and may contribute to disease flare. ${ }^{10}$

Brain-gut psychotherapies, including cognitive-behavior therapy (CBT) and gut-directed hypnotherapy, are optimally delivered by mental health professionals specializing in psychogastroenterology, a field dedicated to applying effective psychological techniques to GI problems. These therapies have the capacity to reduce health care utilization and symptom burden, ${ }^{2,11,12}$ especially when they are integrated directly into GI practice settings. ${ }^{9,13,14}$ Specifically, brain-gut psychotherapies work on 2 related pathways-they target abdominal pain, visceral hypersensitivity, and GI motility; and/or facilitate improved coping, resilience, and selfregulation skills. ${ }^{11}$ Without a gastroenterologist's strong, compelling recommendation for these effective therapies and his/her knowledge about how to successfully facilitate referrals for such treatments, many patients do not receive care at all, ${ }^{13}$ or do so too late in the process, when selfmanagement has failed and refractory psychopathology and/or inflammatory disease have developed. ${ }^{2}$ There is also mounting evidence that stress has important and commonly deleterious effects on gut function through neural-, immune-, and microbiome-related interactions. ${ }^{15}$ Table 1 summarizes the best practices for promoting the use of brain-gut psychotherapies in routine GI care for best clinical outcomes.

Best Practice Advice 1: Gastroenterologists should routinely assess health-related quality of life, symptomspecific anxiety, early life adversity, and functional impairment as these relate to a patient's digestive symptoms.

Individuals with GI disorders tend to have significantly impaired quality of life (QOL) compared to the general population, ${ }^{16-18}$ with IBS patients in particular demonstrating lower QOL than patients with end-stage renal disease and diabetes. ${ }^{19}$ Across functional and organic diseases alike, the most pronounced impact of GI symptoms 
Table 1.Best Practice Update: Incorporating Psychogastroenterology Into Management of Digestive Disorders

\begin{tabular}{|c|c|}
\hline Description & $\begin{array}{l}\text { The current review provides the reader with a framework to understand the scientific rationale and best practices } \\
\text { associated with incorporating brain-gut psychotherapies into routine Gl care. We discuss how } \\
\text { gastroenterologists can employ state-of-the-art assessment and referral techniques that ensure a tailored, } \\
\text { precision-medicine behavioral care pathway into their unique practice settings, across the full spectrum of } \\
\text { digestive disease. }\end{array}$ \\
\hline $\begin{array}{l}\text { Methods } \\
\text { Best Practice Advice }\end{array}$ & These practice updates come from review of the literature, including existing systematic reviews and expert opinion. \\
\hline 1 & $\begin{array}{l}\text { Gastroenterologists should routinely assess health-related QOL, symptom-specific anxieties, early life adversity, and } \\
\text { functional impairment related to a patient's digestive symptoms. }\end{array}$ \\
\hline 2 & $\begin{array}{l}\text { Gastroenterologists should master patient-friendly language on the following topics: the brain-gut pathway and how } \\
\text { this pathway can become dysregulated by any number of factors; the psychosocial risk, perpetuating, and } \\
\text { maintaining factors of Gl diseases; and why the gastroenterologist is referring a patient to a mental health } \\
\text { provider. }\end{array}$ \\
\hline 3 & Gastroenterologists should know the structure and core features of the most effective brain-gut psychotherapies. \\
\hline 4 & $\begin{array}{l}\text { Gastroenterologists should establish a direct referral and ongoing communication pathway with 1-2 qualified mental } \\
\text { health providers and assure patients that he or she will remain part of their care team. }\end{array}$ \\
\hline 5 & $\begin{array}{l}\text { Gastroenterologists should familiarize themselves with } 1 \text { or } 2 \text { neuromodulators that can be used to augment } \\
\text { behavioral therapies when necessary. }\end{array}$ \\
\hline
\end{tabular}

on QOL include fatigue, limitation of life activities, and pain. ${ }^{19}$ The impact of GI disorders on QOL, including which domains of QOL are affected, varies greatly among patients with the same diagnosis, even when disease history, location, severity, or medical therapies are similar. QOL is largely dependent on individual differences in coping and resilience, as well as the experience of GI-specific anxiety, need for lifestyle adaptations, and stress. Patients with substantial GI-related life impact may need more, or differently targeted, medical interventions than other patients with the same diagnoses, and routine assessment of life impact and distress associated with GI symptoms could identify patients in need of extra support early on in care.

Assessment of health-related QOL can be informal and brief, using 1-2 open-ended questions that invite the patient to provide the most relevant information about the life impact of the physical symptoms, for example, "How do your bowel symptoms interfere with your ability to do what you want to do in your daily life?" and "What areas of your life are affected most?" These simple questions often elicit valuable information about the particular domains of the patient's life that are most affected by GI problems. There are multiple benefits to this approach:

1. Without an open-ended option, the physician may remain unaware of key areas where targeted medical intervention can be of greatest help for restoring satisfactory life functioning.

2. These questions about life impact build rapport by indicating to patients that their doctor is aware of, and interested in, the burden of their illness.

3. The patients' responses to open-ended queries about life impact of symptoms can also provide useful comparison data points for rechecking later to assess the effectiveness of interventions in improving the domains that are most important to his/her life and well-being (eg, "Did you make it out on the boat this summer?"). This gives a more complete picture of true progress in managing the patient's health problem than does information about changes in frequency or type of digestive tract symptoms alone.

4. Impact questions can identify patients who clearly need help from a behavioral health professionals to cope more effectively with their illness-when a referral to a GI psychologist is proposed specifically to help improve QOL, it is more likely to be well received.

Asking about early life adversity to understand whether the patient had a personal history of physical, emotional, or sexual abuse; witnessed domestic violence in their household growing up; was raised by a caregiver with a substance abuse problem or mental illness; or had a family member who was incarcerated, could identify critical factors affecting the onset and expression of functional GI disorder symptoms ${ }^{20}$ and point out which patients would benefit from psychological intervention earlier on in care. One commonly used screening questionnaire for early life adversity is the Adverse Childhood Experience Questionnaire. ${ }^{21}$

Symptom-specific anxiety can also amplify disease burden and lead to higher health care utilization. ${ }^{22,23} \mathrm{~A}$ survey of 1242 IBS patients ${ }^{24}$ found, for example, that a substantial minority of the patients thought that their disorders could develop into colitis $(43 \%)$ or cancer $(21 \%)$. In IBD, patients are worried about the effect of biologic therapies or surgery; that pain indicates flare, obstruction, or perforation; or about their risk of infertility. ${ }^{25,26}$ These concerns or fears can be uncovered by asking a simple and direct open-ended question: "What worries or concerns do you have about your symptoms?," which can yield an important opportunity for education and reassurance. Eliciting and discussing whatever worries and concerns the patient has about his or her condition can also help identify instances where excessive anxiety, catastrophizing, or depressive symptoms weigh so heavily in the patient's disease experience that a referral to a behavioral health care provider is needed. 
For certain disorders, disease-specific QOL questionnaires (such as the Irritable Bowel Syndrome Quality of Life $^{27}$ and the Inflammatory Bowel Disease Questionnaire ${ }^{28}$ ) can be useful clinical aids for quantifying the current impact of the disease on patients' lives. If it is feasible to incorporate such measures routinely into clinic visits, the scores they provide are another important means of identifying and addressing marked QOL impact of the disease and how it changes in response to interventions. In contrast, general QOL measures like the 36-Item Short-Form Health Survey ${ }^{29}$ are typically not very clinically useful in GI care, as it is unclear whether poor scores are attributable to the GI disorder or something else, and as they are also less likely to reflect treatment response well. Routine screening for depression and anxiety is not required for the general GI population but may be helpful in special, high-risk populations, including IBD. ${ }^{30}$ For some patients who exhibit such symptoms, a quick measurement to assess their clinical significance may be helpful. Brief questionnaires, such as the free-to-use Patient Health Questionnaire- $9^{31}$ for depression, are useful to have on hand for this purpose. The National Institutes of Health-supported PROMIS (Patient-Reported Outcomes Measurement Information System) screening tools are also free, and may be useful when patients are being screened in comparison to the general population (eg, such as in primary care). ${ }^{32}$

Best Practice Advice 2: Gastroenterologists should master patient-friendly language on the following related topics: bi-directional brain-gut pathway and how this pathway can become dysregulated by any number of factors; psychosocial factors that perpetuate or exacerbate GI symptoms regardless of original etiology; and why the gastroenterologist is referring a patient to a mental health provider.

Research has increasingly elucidated the important role of the central nervous system's neural, hormonal, and immunological bi-directional communication with the gut in maintaining normal GI functioning and modulating disease activity. ${ }^{33,34}$ The brain-gut communication pathway is not only an important concept in functional disorders, where it is typically discussed most, but in all GI disorders. Chronic stress and emotional distress impair the ability of the body to control inflammation, ${ }^{35}$ which increases risk of both peripheral and central pain sensitization in the GI in general, and can affect symptom status and clinical outcomes in disorders, including IBD, ${ }^{36}$ gastroesophageal reflux disease, and peptic ulcer disease. ${ }^{37}$ Even though the details are complicated, the basic concept of this brain-gut communication pathway is fairly simple and provides a foundation for understanding many aspects of GI health problems and their treatment. See Table 2 for essential components of a patient-friendly brain-gut explanation.

Educating patients about the brain-gut axis early in the relationship is helpful so that they do not perceive this as being brought up only after everything else has failed, with the implication of solely a psychological cause of symptoms. If, as is often the case, the conventional medications or treatment approach produce an unsatisfactory degree of relief, this framework gives patients a clear rationale for alternative approaches. Psychological interventions, such as CBT or gut-directed hypnosis, make good sense as GI interventions when explained in the context of a dynamic and continually active brain-gut communication pathway. Selfregulatory and self-care methods, such as meditation, relaxation, yoga, and physical exercise, can also be an integral part of GI health. Additionally, the gut-brain pathway can provide an explanation for inflammatory-related mood and stress effects, and the need for neuromodulation to counteract the impact on the brain from GI pathology.

\section{Conceptualizing the Behavioral Factors That Perpetuate or Exacerbate Gastrointestinal Symptoms Regardless of Original Etiology}

Educating patients about behavioral factors that can exacerbate and perpetuate a health problem is often

Table 2. Key Points in Patient Education About the Brain-Gut Axis, in Lay Language

In recent years, we have learned that the brain and the intestines are much more closely connected than was previously thought, and the brain actually plays a big role in keeping the functioning of the intestines normal and healthy.

1. The brain and the gut (intestines) communicate with each other continually through nerves and chemical signals.

2. The intestines send frequent messages to the brain to let the brain know about their condition, such as fullness from a meal or the need to have a bowel movement.

3. The brain usually dampens these nerve signals coming from the intestines so they are not uncomfortable, and keeps them outside our conscious awareness most of the time.

4. The brain sends frequent messages to the intestines, both in response to these internal messages from the gut and also to help tune the activity of gut muscles, secretion of acid and fluids in the gut, and immune activity, to help the intestines coordinate their functioning in the best way according to what is going on inside them and our lives (eg, telling the gut to increase stomach acid secretion when food is expected and instructing the intestines to inhibit its digestive activity during physical exercise).

5. This normal brain-gut communication can sometimes go wrong when something disturbs the brain or the nervous system for an extended time, such as very substantial life stress, strong negative emotions, or inadequate sleep. Inflammation or infection in the body can also make the normal brain-gut communication go awry. When that happens, for any reason, the brain perceives sensations from the gut more strongly than usual and may start sending inappropriate signals down to the gut that disturb intestinal functioning.

Because the brain is a part of the control system of the gut, it is often possible to get it to help reduce the intestinal disturbance and symptoms regardless of whether it is actively contributing to making the symptoms happen. This can be done by specialized psychological treatment, for example, CBT or gut-directed hypnosis, and also with medications that make the brain less sensitive to input from the intestines. 
necessary. A physical GI problem may seem to the average person to be something relatively isolated from the conditions of daily life and outside of his/her control. In reality, however, stress, poor quality and inadequate quantity of sleep, maladaptive eating patterns (like consuming large meals or eating late at night), and lack of physical activity are potent symptom contributors to a range of health problems-behaviorally, these perpetuating and maintaining factors are within the patient's control and can be adjusted. It should also be emphasized with patients that even though these and other behavioral and psychosocial contributing factors may exacerbate symptoms and can be changed to attenuate the disease severity, they are generally not the causes of the GI disease. It is also helpful to remind patients that most health problems are multifactorial and that there is unlikely to be a single solution, at least long-term. As a result, introducing the concept of routine referral to a mental health care provider early in the evaluation will be better received. Similarly, factors that initiate a disease process (inflammation or immune dysregulation, surgery, stress) do not always remain active, despite ongoing symptoms. Models where this can be illustrated well include post-infectious IBS and IBD with functional overlap, both common conditions with etiologies that differ from their maintaining factors. ${ }^{38,39}$ Highlighting these models can allow patients to move past looking for a cause or explanation for why they are symptomatic (eg, infection they got, surgery they had) toward solutions that they can act upon (eg, diet, lifestyle, stress, adherence).

\section{Why the Gastroenterologist Is Referring a Patient to a Mental Health Provider}

One of the most difficult parts of providing behavioral medicine treatments for patients presenting with GI complaints in the outpatient office is helping the patients to understand why they are being referred to a psychologist for a GI symptom. If this is not done correctly, many patients will believe the physician is discounting their physical symptoms and believes that the patient's symptoms are imagined or all psychologically based. Failure to properly explain the reason for referral will lead to poor follow through and will likely lead the patient to seek care with another provider. Furthermore, this failure can negatively impact what is an otherwise good physician-patient relationship, and decrease the likelihood of successful symptom control. It is also important to recognize that, as the physician, you do not need to decide on the specific type of behavior therapy the patient will benefit from-this is usually best decided by the behavioral health care provider. See Table 3 for key patient education points for accomplishing effective referral to a psychologist.
Gl psychologists are licensed providers with a doctoral degree in their profession who fall under the American Psychological Association's recognized subspecialty of clinical health psychology, an area of psychology devoted to the application of psychological principles to medical conditions and to the integration of psychologists into medical settings and multidisciplinary teams. Health psychologists can develop further expertise working within a specific medical subspecialty (Gl, obstetrics/gynecology, oncology, pain) or practice as generalists. Generalists are often pleased to see patients with Gl conditions, and will usually seek out either specialized training in Gl or read the literature on the disease they are treating and its effective treatment approaches. Psychiatrists are medical doctors focused on determining a patient's specific psychiatric diagnosis and remediating symptoms with psychotropic medication. A smaller subset of psychiatrists specialize in psychosomatic medicine and are a welcome addition to a multidisciplinary Gl team. The Rome Foundation has recently created an internationally based psychogastroenterology section that brings together health care professionals with clinical and/or research interests in this growing subspecialty-psychologists, gastroenterologists, psychiatrists, internists/pediatricians with psychosomatic interests, as well as non-doctorally trained providers such as nurses, nurse practitioners, physician assistants and social workers, to facilitate best clinical and research practices in this evolving field. Future directions in this area may include establishing core competencies or necessary training credentials for the practice of psychogastroenterology.

Best Practice Advice 3: Gastroenterologists should know the structure and core features of the most effective brain-gut psychotherapies.

Brain-gut psychotherapies are those that leverage the brain's ability to bring under voluntary control those symptom processes that seem, initially, to be driven completely by the gut. Brain-gut psychotherapies share several important features that distinguish them from traditional psychotherapy approaches-they are typically short-term and GI symptom-focused; and patients with comorbid chronic depression or anxiety are typically not good candidates. These therapies offer the best results when delivered by a health psychologist or other medically trained mental health provider who is familiar with the physiological basis of symptoms and the goals of the medical team. Brain-gut therapies are skills-based and focus specifically on the down-regulation of unpleasant GI sensations, decreasing avoidance behaviors associated with fear of having symptoms and building coping and resilience to stress or lifestyle changes imposed by a chronic condition. These therapies differ from more traditional forms of psychotherapy, which focus on providing support, normalizing psychopathology, and/or facilitating insight into the reason the patient is experiencing symptoms. Another benefit of these skills-based therapies is that their impact can be durable long after therapy is discontinued, making 
Table 3. Key Points in Patient Education About a Referral to a Mental Health Provider, From the Perspective of a Gastroenterologist, in Lay Language

Once the patient understands the brain-gut connection, you can shift attention to the therapies that work on this pathway.

1. Introduce the concept of neuromodulation and discuss how medications. such as low-dose antidepressants and anti-seizure medications, can alter neurologic transmission of signals to the brain and dampen some of the discomfort they are feeling.

- Cons of the medications including their side effects; limited efficacy, especially when compared to behavioral therapies; and that most patients will need to commit to these medications long-term.

2. When introducing the concept of the patient undergoing hypnosis or cognitive therapy, bring up a few examples to help the patient understand the biologic reasoning behind the treatments.

- One way to describe the connection is to discuss how their Gl tract is always moving through peristalsis and accommodation, but the brain's normal job is to prevent you from feeling this movement because feeling your Gl tract all day would be too distracting. You can compare this to putting on a watch or your glasses in that you feel it when you put them on to make sure they are correctly placed, but after a minute or so your brain will not pay attention to this signal-this is a healthy mechanism the brain has developed so that you can focus your attention on important tasks at hand.

- This process of filtering out non-essential sensations is extremely important but very complex and poorly understood. Psychological therapies can help you divert your brain's attention away from the symptoms, can help you to be less bothered by your symptoms and allow you to focus on more important things.

3. Stay basic in terms of the effects and potential mechanisms of brain-gut psychotherapies. This basic information sets the stage for a much more informed visit with the psychologist and dispels the pretense that the Gl doctor thinks that the patient has nothing wrong.

- You can say that CBT provides patients with a skill set that helps them change the way they view their symptoms, or to make their symptoms feel less threatening.

- Hypnosis is not like a stage entertainment show and is nothing magical-it is a real medical therapy that has been studied with great outcomes.

- There are no side effects to these therapies, and the positive effects on symptoms often last for years.

them cost-effective over the long-term when compared to psychotropics. ${ }^{40}$ For a recent review of brain-gut psychotherapies in IBS and IBD, see Ballou and Keefer. ${ }^{11}$ IBS patients in particular often appreciate hearing that there are more than 30 randomized controlled trials for CBT and 11 for hypnosis in IBS alone, with numbers needed to treat between 2 and $4 .^{12}$ These therapies have also been used successfully across a spectrum of digestive diseases, including non-cardiac chest pain, functional dyspepsia, gastroesophageal reflux disease, and IBD.

CBT is one of the most robust, well-tested, brain-gut psychotherapies, with more than 30 randomized controlled trials and a number needed to treat of $3 .^{12} \mathrm{CBT}$ focuses on remediating an individual's maladaptive or "unhelpful" thoughts, feelings, and behaviors as they relate to any number of symptoms, rather than identifying a root cause or initiating event. CBT has been used successfully for depressed teenagers with IBD, ${ }^{41-44}$ and as a way to augment self-management behavior for patients with Crohn's disease. $^{45}$ It has been effectively delivered by nurses, ${ }^{46}$ over the telephone, ${ }^{47}$ and in groups. ${ }^{48}$ CBT for functional GI disorders, including those seen in the context of IBD, targets the specific maladaptive cognitive-affective processes known to impact GI symptom experience at the level of the brain $^{49}$-these include catastrophizing, cognitive inflexibility, fear of symptoms, or hypervigilance/attentional bias to benign gut sensations. Depending on a patient's GI symptom profile, various forms of skills remediation can be used, including diaphragmatic breathing, cognitive restructuring, or learning new problem-solving skills. CBT for IBS can now be delivered with efficacy equivalent to longer treatment courses, in as few as 4 therapy sessions, ${ }^{50}$ and has shown promise when delivered online for both IBS and IBD. $^{51-54}$ Elements of CBT, particularly diaphragmatic breathing, have been used for esophageal conditions, including rumination disorder and supragastric belching. ${ }^{14,55}$ Building up a tolerance to unpleasant GI symptoms through interoceptive exposure, another CBT technique, with and without mindfulness, ${ }^{53,56,57}$ has also been used successfully in patients with symptom-specific anxiety. ${ }^{58}$

Gut-directed hypnotherapy is also a well-tested therapy for a range of pediatric and adult GI disorders, including IBS ( $>11$ randomized controlled trials), heartburn, and IBD. ${ }^{59}$ Targets of hypnotherapy include visceral hypersensitivity, motility disturbance, hypervigilance to benign sensations, and somatization. Two protocols in particular, the 7-session North Carolina Protocol ${ }^{60}$ and the 12-session Manchester protocol ${ }^{61}$ have demonstrated similar benefits in refractory IBS, with up to $80 \%$ of patients acquiring at least a $50 \%$ reduction in IBS Severity Scoring. ${ }^{62,63}$ The fully scripted North Carolina Protocol has been delivered as a home-based therapy ${ }^{64}$ and has also been validated for use with gastroesophageal reflux ${ }^{65}$ and ulcerative colitis, ${ }^{66}$ and is particularly useful for enabling less-experienced providers to successfully deliver gut-directed hypnotherapy. ${ }^{67}$

Best Practice Advice 4: Gastroenterologists should establish a direct referral and ongoing communication pathway with 1-2 qualified mental health providers and assure patients that he or she will remain part of their care team.

Access to evidence-based brain-gut psychotherapies limits their utility and, while not every gastroenterologist will know of a GI health psychologist, a viable alternative is to reach out to the state psychological society (eg, [state name] Psychological Association) or to a national registry for the names of therapists who are interested in seeing GI patients and who are familiar with at least one of the evidence-based brain-gut psychotherapies. The typical toolbox of someone equipped to see GI patients include experience with medical populations or chronic illness; 
collaborates with physicians; has been trained under a cognitive-behavioral theoretical orientation; has a collaborative, active patient interaction style; practices 1 or more of the evidence-based brain-gut psychotherapies; and, if he or she practices hypnotherapy, their training was provided through a reputable organization focused on training health professionals. Having a clear referral and communication pathway improves outcomes by establishing buy-in on the part of the patient (they are not just left to find a mental health provider on their own), and ensures that the focus of treatment will be the GI condition. The mental health provider should feel free to call and ask the GI doctor questions around the patient's symptoms, workup, and medical recommendations. GI doctors should expect to receive a copy of the patient's psychological intake report and possibly a final treatment summary. The GI doctors should also plan to see the patient themselves sometime during the course of active psychotherapy as a way to maintain continuity of care, reinforce therapy gains, change or reduce medication use, and troubleshoot any challenges.

Contrary to what many people assume, the presence of significant psychological distress does not suggest suitability for referral to a GI psychologist. Research has shown that many of the brain-gut psychotherapies are less effective when a patient has comorbid psychopathology. ${ }^{68}$ If GI patients have comorbid Axis I disorders and suffer from very pronounced emotional symptoms, it may be best to have those problems addressed first, either by a more general psychologist in the community who focuses on depression and anxiety disorders, or by means of psychotropic medications, as this will often improve the odds of the patients subsequently benefitting from GI-focused psychological treatment. For patients with more moderate emotional symptoms, however, the affective disorders can be addressed in parallel with the work of the GI psychologist. Along these lines, it is helpful for gastroenterologists to have information on a wide range of mental health resources, including free or sliding-scale programs, in their communities to ensure appropriate mental health system access for these patients.

Best Practice Advice 5: Gastroenterologists should familiarize themselves with 1 or 2 neuromodulators that can complement behavioral therapies when necessary.

Neuromodulation/psychotropic use is sometimes necessary and often effective in chronic digestive diseases ${ }^{2}$ and should be on the radar of practicing gastroenterologists, especially given a shortage of qualified psychiatrists. In keeping with the patient-friendly, de-stigmatizing rationale for brain-gut psychotherapies, there may be scenarios in which a health psychologist and gastroenterologist are comfortable that the introduction of a neuromodulator,

Table 4. List of Readings to Enhance Understanding of Best Practice Advice

Recommended source

Drossman DA. Abuse, trauma, and GI illness: is there a link? Am J Gastroenterol 2011;106:14-25.

Drossman, DA. 2012 David Sun Lecture. Helping your patient by helping yourself. How to improve the patient physician relationship by optimizing communication skills. Am J Gastroenterol 2012;106:521-528

Van Oudenhove L, Crowell MD, Drossman DA, et al. biopsychosocial aspects of functional gastrointestinal disorders. Gastroenterology 2016;150:1355-1367.e2.

Mayer EA. Gut feelings: the emerging biology of gut-brain communication. Nat Rev Neurosci 2011;12:453-466.

Kinsinger SW. Cognitive-behavioral therapy for patients with irritable bowel syndrome: current insights. Psychol Res Behav Manag 2017;10:231-237.

Palsson OS. Hypnosis treatment of gastrointestinal disorders: a comprehensive review of the empirical evidence. Am J Clin Hypn 2015;58:134-158.

Ballou S, Keefer L. Psychological interventions for irritable bowel syndrome and inflammatory bowel diseases. Clin Transl Gastroenterol 2017;8:e214

Riehl ME, Kinsinger S, Kahrilas PJ, et al. Role of a health psychologist in the management of functional esophageal complaints. Dis Esophagus 2015;28:428-436.

Kinsinger SW, Ballou S, Keefer L. Snapshot of an integrated psychosocial gastroenterology service. World J Gastroenterol 2015;21:1893-1899.

Sobin WH, Heinrich TW, Drossman DA. Central neuromodulators for treating functional Gl disorders: a primer. Am J Gastroenterol 2017;112:693-702.

Thorkelson G, Bielefeldt K, Szigethy E. Empirically supported use of psychiatric medications in adolescents and adults with IBD. Inflamm Bowel Dis 2016;22:1509-1522.

Evidence-based trade books for patients with IBS

Lackner JM. Controlling IBS the Drug-Free Way: A 10-Step Plan for Symptom Relief. New York: Harry Abrams Publisher, 2007. ISBN \#978-1584795759.

Barney P, Weisman P, Jarrett M, et al. Master Your IBS: An 8-Week Plan Proven to Control the Symptoms of Irritable Bowel Syndrome. Bethesda, MD: AGA Press, 2010. ISBN: 978-1603560092.

Mayer EA. The Mind-Gut Connection: How the Hidden Conversation Within Our Bodies Impacts Our Mood, Our Choices, and Our Overall Health. New York: Harper Wave, 2016. ISBN: 978-0062376558.
Associated BPA 
particularly a selective serotonin reuptake inhibitor; serotonin and norepinephrine reuptake inhibitor; or tricyclic antidepressant, could augment ongoing behavior therapy. Common reasons include a diagnosis of a minor comorbid depression or anxiety disorder, lack of insight or motivation that precludes participation in in psychological treatment, or chronic pain that is not responding fully to behavioral intervention. See Sobin et $\mathrm{al}^{69}$ for a primer in central neuromodulation for functional GI disorders and Thorkelson et $\mathrm{al}^{70}$ for similar information in IBD.

\section{Summary and Future Directions}

The importance of the gastroenterologist in the promotion of psychogastroenterology cannot be overstated. We have discussed best practices for the assessment and referral of patients across the spectrum of disease to braingut psychotherapies, including CBT and gut-directed hypnotherapy. There are research and clinical gaps that will need to be addressed in order to foster widespread integration of these services. From a research perspective, gaps include lack of comparative effectiveness trials that compare brain-gut psychotherapies against each other and/ or against psychotropic medications and lack of sufficient research to date of other promising brain-gut therapies, such as mindfulness meditation ${ }^{71}$ or acceptance-based approaches. ${ }^{72}$ There are only a few studies supporting the impact of brain-gut psychotherapies on actual disease course or inflammation in conditions such as Crohn's disease and ulcerative colitis, ${ }^{6,73,74}$ and limited studies demonstrating the utility of brain-gut psychotherapies in accelerating or enhancing the efficacy of pharmacologic therapies. Clinical gaps include need for better coverage for these therapies by insurance-health psychologists can be reimbursed for health and behavior codes for treating these conditions (Current Procedural Terminology codes 96150/ 96152), but there are restrictions on which other types of professionals can use them. Medicare and commercial insurance plans often cover the cost of services, but many providers are out of network or do not accept insurance. It is sometimes helpful to remind patients, however, that these highly effective therapies are short term (usually less than 3 months) and have a one-time total cost between $\$ 1000$ and $\$ 2000$ out of pocket. The effects of these treatments are commonly durable for several years and therapy usually does not need to be re-administered. If you point out to patients that what they pay out of pocket per month for other generally less effective uncovered interventions, such as probiotics, herbs, special diets, colonic cleanses, then the out-of-pocket expense often becomes of less concern. Research demonstrating the cost-effectiveness of these therapies is underway and may lead to wider adoption of these services by accountable care organizations, payors, employers, and other stakeholders. Lastly, there is a need for more trained providers and the expansion of remote, online, or digitally based brain-gut therapies that could offset overhead and other therapy costs. For a list of additional readings in this important area, please see Table 4.

\section{References}

1. Peery AF, Crockett SD, Barritt AS, et al. Burden of gastrointestinal, liver, and pancreatic diseases in the United States. Gastroenterology 2015;149:1731-1741.e3.

2. Van Oudenhove L, Crowell MD, Drossman DA, et al. Biopsychosocial aspects of functional gastrointestinal disorders. Gastroenterology 2016;150:1355-1367.e2.

3. Yadlapati R, Dakhoul L, Pandolfino JE, et al. The quality of care for gastroesophageal reflux disease. Dig Dis Sci 2017;62:569-576.

4. Roman S, Keefer L, Imam H, et al. Majority of symptoms in esophageal reflux PPI non-responders are not related to reflux. Neurogastroenterol Motil 2015;27:1667-1674.

5. Drossman DA, Chang L, Bellamy N, et al. Severity in irritable bowel syndrome: a Rome Foundation Working Team report. Am J Gastroenterol 2011;106:1749-1759; quiz 1760.

6. Drossman D, Szigethy E. The narcotic bowel syndrome: a recent update. Am J Gastroenterol Suppl 2014;2: 22-30.

7. Keefer L, Drossman DA, Guthrie E, et al. Centrally mediated disorders of gastrointestinal pain. Gastroenterology 2016;150:1406-1419.

8. Click B, Ramos Rivers C, Koutroubakis IE, et al. Demographic and clinical predictors of high healthcare use in patients with inflammatory bowel disease. Inflamm Bowel Dis 2016;22:1442-1449.

9. Regueiro MD, McAnallen SE, Greer JB, et al. The inflammatory bowel disease specialty medical home: a new model of patient-centered care. Inflamm Bowel Dis 2016;22:1971-1980.

10. Keefer L, Kane SV. Considering the bidirectional pathways between depression and IBD: recommendations for comprehensive IBD care. Gastroenterol Hepatol (N Y) 2017;13:164-169.

11. Ballou S, Keefer L. Psychological interventions for irritable bowel syndrome and inflammatory bowel diseases. Clin Transl Gastroenterol 2017;8:e214.

12. Ford AC, Quigley EM, Lacy BE, et al. Effect of antidepressants and psychological therapies, including hypnotherapy, in irritable bowel syndrome: systematic review and meta-analysis. Am J Gastroenterol 2014; 109:1350-1365; quiz 1366.

13. Kinsinger SW, Ballou S, Keefer L. Snapshot of an integrated psychosocial gastroenterology service. World J Gastroenterol 2015;21:1893-1899.

14. Riehl ME, Kinsinger S, Kahrilas PJ, et al. Role of a health psychologist in the management of functional esophageal complaints. Dis Esophagus 2015;28:428-436.

15. Bonaz BL, Bernstein CN. Brain-gut interactions in inflammatory bowel disease. Gastroenterology 2013; 144:36-49.

16. Koloski NA, Talley NJ, Boyce PM. The impact of functional gastrointestinal disorders on quality of life. Am J Gastroenterol 2000;95:67-71.

17. Strine TW, Chapman DP, Flowers N. Psychological distress and impaired quality of life common among community-dwelling adults with lower gastrointestinal disorders. Dig Dis Sci 2007;52:70-77. 
18. Bernklev T, Jahnsen J, Lygren I, et al. Health-related quality of life in patients with inflammatory bowel disease measured with the Short Form-36: psychometric assessments and a comparison with general population norms. Inflamm Bowel Dis 2005;11:909-918.

19. Gralnek IM, Hays RD, Kilbourne A, et al. The impact of irritable bowel syndrome on health-related quality of life. Gastroenterology 2000;119:654-660.

20. Park SH, Naliboff BD, Shih W, et al. Resilience is decreased in irritable bowel syndrome and associated with symptoms and cortisol response. Neurogastroenterol Motil 2018;30(1).

21. Centers for Disease Control and Prevention. Behavioral Risk Factor Surveillance System Survey Questionnaire: ACE Module. Atlanta, GA: US Department of Health and Human Services, 2010.

22. Jerndal P, Ringstrom G, Agerforz P, et al. Gastrointestinal-specific anxiety: an important factor for severity of GI symptoms and quality of life in IBS. Neurogastroenterol Motil 2010;22:646-e179.

23. Labus JS, Mayer EA, Chang L, et al. The central role of gastrointestinal-specific anxiety in irritable bowel syndrome: further validation of the visceral sensitivity index. Psychosom Med 2007;69:89-98.

24. Halpert A, Dalton CB, Palsson O, et al. What patients know about irritable bowel syndrome (IBS) and what they would like to know. National Survey on Patient Educational Needs in IBS and development and validation of the Patient Educational Needs Questionnaire (PEQ). Am J Gastroenterol 2007;102:1972-1982.

25. Keeton RL, Mikocka-Walus A, Andrews JM. Concerns and worries in people living with inflammatory bowel disease (IBD): a mixed methods study. J Psychosom Res 2015;78:573-578.

26. Stjernman H, Tysk C, Almer S, et al. Worries and concerns in a large unselected cohort of patients with Crohn's disease. Scand J Gastroenterol 2010;45: 696-706.

27. Patrick DL, Drossman DA, Frederick IO, et al. Quality of life in persons with irritable bowel syndrome: development and validation of a new measure. Dig Dis Sci 1998; 43:400-411.

28. Irvine EJ. Development and subsequent refinement of the inflammatory bowel disease questionnaire: a quality-oflife instrument for adult patients with inflammatory bowel disease. J Pediatr Gastroenterol Nutr 1999; 28:S23-S27.

29. McHorney CA, Ware JE Jr, Raczek AE. The MOS 36-Item Short-Form Health Survey (SF-36): II. Psychometric and clinical tests of validity in measuring physical and mental health constructs. Med Care 1993;31:247-263.

30. Farraye FA, Melmed GY, Lichtenstein GR, et al. ACG clinical guideline: preventive care in inflammatory bowel disease. Am J Gastroenterol 2017;112:241-258.

31. Kroenke K, Spitzer RL, Williams JB. The PHQ-9: validity of a brief depression severity measure. J Gen Intern Med 2001;16:606-613.

32. Kim J, Chung H, Askew RL, et al. Translating CESD-20 and PHQ-9 Scores to PROMIS Depression. Assessment 2017;24:300-307.
33. Dinan TG, Cryan JF. The microbiome-gut-brain axis in health and disease. Gastroenterol Clin North Am 2017; 46:77-89.

34. Al Omran Y, Aziz Q. The brain-gut axis in health and disease. Adv Exp Med Biol 2014;817:135-153.

35. Cohen S, Janicki-Deverts D, Doyle WJ, et al. Chronic stress, glucocorticoid receptor resistance, inflammation, and disease risk. Proc Natl Acad Sci U S A 2012; 109:5995-5999.

36. Bonaz B. Inflammatory bowel diseases: a dysfunction of brain-gut interactions? Minerva Gastroenterol Dietol 2013;59:241-259.

37. Nardone G, Compare D. The psyche and gastric functions. Dig Dis 2014;32:206-212.

38. Schwille-Kiuntke J, Mazurak N, Enck P. Systematic review with meta-analysis: post-infectious irritable bowel syndrome after travellers' diarrhoea. Aliment Pharmacol Ther 2015;41:1029-1037.

39. Hoekman DR, Zeevenhooven J, D'Haens GR, et al. The prevalence of irritable bowel syndrome-type symptoms in inflammatory bowel disease patients in remission. Eur J Gastroenterol Hepatol 2017;29:1086-1090.

40. Creed F, Fernandes L, Guthrie E, et al. The costeffectiveness of psychotherapy and paroxetine for severe irritable bowel syndrome. Gastroenterology 2003; 124:303-317.

41. Szigethy E, Kenney E, Carpenter J, et al. Cognitivebehavioral therapy for adolescents with inflammatory bowel disease and subsyndromal depression. J Am Acad Child Adolesc Psychiatry 2007;46:1290-1298.

42. Szigethy E, Whitton SW, Levy-Warren A, et al. Cognitivebehavioral therapy for depression in adolescents with inflammatory bowel disease: a pilot study. J Am Acad Child Adolesc Psychiatry 2004;43:1469-1477.

43. Thompson RD, Craig A, Crawford EA, et al. Longitudinal results of cognitive behavioral treatment for youths with inflammatory bowel disease and depressive symptoms. J Clin Psychol Med Settings 2012;19:329-337.

44. van den Brink G, Stapersma L, El Marroun $H$, et al. Effectiveness of disease-specific cognitive-behavioural therapy on depression, anxiety, quality of life and the clinical course of disease in adolescents with inflammatory bowel disease: study protocol of a multicentre randomised controlled trial (HAPPY-IBD). BMJ Open Gastroenterol 2016;3:e000071.

45. Keefer L, Doerfler B, Artz C. Optimizing management of Crohn's disease within a project management framework: results of a pilot study. Inflamm Bowel Dis 2012; 18:254-260.

46. Heitkemper MM, Jarrett ME, Levy RL, et al. Selfmanagement for women with irritable bowel syndrome. Clin Gastroenterol Hepatol 2004;2:585-596.

47. Jarrett ME, Cain KC, Burr RL, et al. Comprehensive selfmanagement for irritable bowel syndrome: randomized trial of in-person vs. combined in-person and telephone sessions. Am J Gastroenterol 2009;104:3004-3014.

48. Blanchard EB, Lackner JM, Sanders K, et al. A controlled evaluation of group cognitive therapy in the treatment of irritable bowel syndrome. Behav Res Ther 2007;45: 633-648. 
49. Naliboff BD, Mayer EA. Brain imaging in IBS: drawing the line between cognitive and non-cognitive processes. Gastroenterology 2006;130:267-270.

50. Lackner JM, Jaccard J, Krasner SS, et al. Selfadministered cognitive behavior therapy for moderate to severe irritable bowel syndrome: clinical efficacy, tolerability, feasibility. Clin Gastroenterol Hepatol 2008; 6:899-906.

51. Mikocka-Walus A, Bampton P, Hetzel D, et al. Cognitivebehavioural therapy for inflammatory bowel disease: 24-month data from a randomised controlled trial. Int $\mathrm{J}$ Behav Med 2017;24:127-135.

52. Bonnert M, Olen O, Lalouni M, et al. Internet-delivered cognitive behavior therapy for adolescents with irritable bowel syndrome: a randomized controlled trial. Am J Gastroenterol 2017;112:152-162.

53. Ljotsson B, Andersson G, Andersson E, et al. Acceptability, effectiveness, and cost-effectiveness of internetbased exposure treatment for irritable bowel syndrome in a clinical sample: a randomized controlled trial. BMC Gastroenterol 2011;11:110.

54. Ljotsson B, Hedman E, Andersson E, et al. Internetdelivered exposure-based treatment vs. stress management for irritable bowel syndrome: a randomized trial. Am J Gastroenterol 2011;106:1481-1491.

55. Halland M, Parthasarathy G, Bharucha AE, et al. Diaphragmatic breathing for rumination syndrome: efficacy and mechanisms of action. Neurogastroenterol Motil 2016;28:384-391.

56. Ljotsson B, Falk L, Vesterlund AW, et al. Internetdelivered exposure and mindfulness based therapy for irritable bowel syndrome-a randomized controlled trial. Behav Res Ther 2010;48:531-539.

57. Gaylord SA, Whitehead WE, Coble RS, et al. Mindfulness for irritable bowel syndrome: protocol development for a controlled clinical trial. BMC Complement Altern Med 2009;9:24.

58. Craske MG, Wolitzky-Taylor KB, Labus J, et al. A cognitive-behavioral treatment for irritable bowel syndrome using interoceptive exposure to visceral sensations. Behav Res Ther 2011;49:413-421.

59. Palsson OS. Hypnosis treatment of gastrointestinal disorders: a comprehensive review of the empirical evidence. Am J Clin Hypn 2015;58:134-158.

60. Palsson OS. Standardized hypnosis treatment for irritable bowel syndrome: the North Carolina protocol. Int J Clin Exp Hypn 2006;54:51-64.

61. Whorwell PJ. Use of hypnotherapy in gastrointestinal disease. Br J Hosp Med 1991;45:27-29.

62. Whorwell PJ, Prior A, Faragher EB. Controlled trial of hypnotherapy in the treatment of severe refractory irritable-bowel syndrome. Lancet 1984;2:1232-1234.
63. Palsson OS, Turner MJ, Johnson DA, et al. Hypnosis treatment for severe irritable bowel syndrome: investigation of mechanism and effects on symptoms. Dig Dis Sci 2002;47:2605-2614.

64. Palsson OS, Turner MJ, Whitehead WE. Hypnosis home treatment for irritable bowel syndrome: a pilot study. Int J Clin Exp Hypn 2006;54:85-99.

65. Riehl ME, Pandolfino JE, Palsson OS, et al. Feasibility and acceptability of esophageal-directed hypnotherapy for functional heartburn. Dis Esophagus 2016;29: 490-496.

66. Keefer L, Taft TH, Kiebles JL, et al. Gut-directed hypnotherapy significantly augments clinical remission in quiescent ulcerative colitis. Aliment Pharmacol Ther 2013;38:761-771.

67. Palsson OS, van Tilburg M. Hypnosis and guided imagery treatment for gastrointestinal disorders: experience with scripted protocols developed at the University of North Carolina. Am J Clin Hypn 2015;58:5-21.

68. Lackner JM, Jaccard J, Krasner SS, et al. How does cognitive behavior therapy for irritable bowel syndrome work? A mediational analysis of a randomized clinical trial. Gastroenterology 2007;133:433-444.

69. Sobin WH, Heinrich TW, Drossman DA. Central neuromodulators for treating functional GI disorders: a primer. Am J Gastroenterol 2017;112:693-702.

70. Thorkelson G, Bielefeldt K, Szigethy E. Empirically supported use of psychiatric medications in adolescents and adults with IBD. Inflamm Bowel Dis 2016;22:1509-1522.

71. Aucoin M, Lalonde-Parsi MJ, Cooley K. Mindfulnessbased therapies in the treatment of functional gastrointestinal disorders: a meta-analysis. Evid Based Complement Alternat Med 2014;2014:140724.

72. Sebastian Sanchez B, Gil Roales-Nieto J, Ferreira NB, et al. New psychological therapies for irritable bowel syndrome: mindfulness, acceptance and commitment therapy (ACT). Rev Esp Enferm Dig 2017:109.

73. Mawdsley JE, Jenkins DG, Macey MG, et al. The effect of hypnosis on systemic and rectal mucosal measures of inflammation in ulcerative colitis. Am J Gastroenterol 2008;103:1460-1469.

74. Szigethy E. Hypnotherapy for inflammatory bowel disease across the lifespan. Am J Clin Hypn 2015;58:81-99.

\section{Received September 29, 2017. Accepted January 11, 2018.}

Reprint requests

Address requests for reprints to: Laurie Keefer, PhD, Division of Gastroenterology, Icahn School of Medicine at Mount Sinai, New York, New York 10029. e-mail: laurie.keefer@mssm.edu; fax: (646) 537-8921.

Conflicts of interest

The authors disclose no conflicts. 